\title{
Gold Nanoparticle in the Application of Forensic Fingermark Development: A Short Review
}

ISSN: 2578-0042

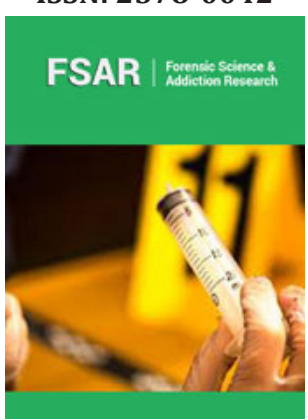

*Corresponding author: Revathi Rajan, Department of Diagnostics and Allied Health Sciences, Malaysia

Submission: 眥 February 15, 2019

Published: 佂March 29, 2019

Volume 4 - Issue 4

How to cite this article: Revathi Rajan, Gold Nanoparticle in the Application of Forensic Fingermark Development: A Short Review. Forensic Sci Add Res. 4(4). FSAR.000596.2019.

DOI: 10.31031/FSAR.2019.04.000596

Copyright@ Revathi Rajan, This article is distributed under the terms of the Creative Commons Attribution 4.0 International License, which permits unrestricted use and redistribution provided that the original author and source are credited.

\author{
Revathi Rajan ${ }^{1,2 *}$, Yusmazura Zakaria ${ }^{2}$, Shaharum Shamsuddin ${ }^{2}$ and Nik \\ Fakhuruddin Nik Hassan² \\ ${ }^{1}$ Department of Diagnostics and Allied Health Sciences, Management \& Science University, \\ Malaysia \\ ${ }^{2}$ School of Health Sciences, University Sains Malaysia, Malaysia
}

\begin{abstract}
The lack of fundamental understanding about the specific reaction behind the attraction of gold nanoparticle to fingermark residue has been reiterated many times over the years. Numerous scientific works has discussed the theoretical possibilities of interaction pathways, mostly favoring electrostatic interaction between negatively charged citrate capped gold nanoparticle and n-terminus of the amino acid in fingermark residue. Nevertheless, in light of recent findings, formation of chemical bonding between the gold nanoparticle and fingermark residue offers plausible explanation.
\end{abstract}

\section{Introduction}

The development of fingermarks using gold nanoparticle (GNPs) was first introduced by Saunders in 1989 [1]. The technique involved two major phases, depositing the citrate capped GNP on the fingermark residue and followed the enhancing step using silver physical developer [1]. Postulation have been made regarding the reaction mechanism by which the citrate capped GNPs are attracted to the fingermark residues. The most popular theory was of electrostatic attraction between the negatively charged citrate ions and the positively charged $\mathrm{N}$-terminus of amino acids present in the fingermark residue [2]. The GNPs, in turn, serve as the nucleation site for the growth of the silver physical developer giving clearer ridge details [3]. This technique was termed multi metal deposition technique because two distinct metals were utilised in the course of the fingermark development [4].

This technique presented several technical drawbacks though. The major limitation of the method includes the stringent reagent synthesis protocol1, requires specially trained personnel and also very narrow margin for error during development process which can drastically change the outcome $[5,6]$. Since the outcome of this method fluctuates even in carefully controlled laboratory conditions, application in the field becomes out of the question [7]. The limitation of this method and the researches that have been done to counteract the disadvantages are discussed in this paper.

\section{Cleanliness of laboratory wares and pre-cautions during reagent synthesis [1]}

The successful synthesis of reagent without aggregation of the particles relies heavily on the use of siliconised glassware and bi-distilled water for all processes. Failure to comply with this rule will result in the creation of unwanted nucleation sites which is detrimental to the synthesis of colloidal gold [1]. Findings have been reported that no significant difference was observed when siliconised glassware were utilised during the process of fingermark development [2], however, cleanliness of glassware is very important during the synthesis of colloidal gold solution.

\section{Physical characteristics of the reagents used in the original MMD method}

The reagents used for the MMD method are being researched to improve the limitation of the original method [1,2]. Each property of the reagent and the method by which the reagent was synthesized contributes hugely to the sensitivity of the reagent, clearer ridge details and also contrast of the developed mark against the background. Modifications were proposed to the preparation of the reagents and also the physical characteristics of the reagents to obtain 
better sensitivity and enhanced resolution of the MMD method $[1,2]$. Among the proposed modifications were homogenously distributed finer nanoparticles, concentration of colloidal gold solution and Tween 20 solution and maintaining $\mathrm{pH}$ and temperature during the development process [1]. Nevertheless, the low contrast of the developed fingermarks against dark or colored background remains a problem even with this modification.

\section{Lower contrast of the developed fingermarks against dark or colored background}

Stauffer et al. [8] attempted to resolve this problem by replacing the silver physical enhancer with gold solution [8]. Since only one metal is used in this process the technique was termed single metal deposition (SMD) method [2,8]. In this paper, the silver physical developer was replaced with tetrachloroauric acid mixed in hydroxylamine hydrochloride solution. This solution functions as a growth medium for the existing seed particle on the fingermark deposit, whereby instantaneous reduction takes place when seed particles are introduced into the solution [9]. The advantages of the this technique was production of lighter colored fingermark, reagent with longer shelf life and lower in cost consumption in comparison to the preceding MMD technique [8].

In order to alleviate the contrast issue, Becue et al. came up with the technique to synthesise thiolated cyclodextrin (CD) couple GNPs. The cyclodextrin has a three-dimensional truncated cone structure that can be exploited to incorporate dye substrate into its cavities. Using the three steps immersion process, dark blue prints were visualised under ambient lighting and was presented with good contrast [7]. Zinc oxide was also used as the enhancer as the replacement for silver physical developer which produced fingermark with luminescent capability under UV-exciting light source. The application of the zinc oxide produced marks with lesser background staining [5]. Both the modified MMD method had the similar working principle as the original MMD method [8]. Another SMD technique was introduced using glucose stabilised GNPs to develop the fingermark. The crucial advantage that this method introduced was the significant reduction in the number of baths during the development process from six steps in MMD method to only three [10]. The resolution of the fingermark's ridge details were also superior to the conventional MMD technique [10].

\section{Sensitivity of the technique}

The sensitivity of the MMD heavily relies on the interaction between the GNPs in the colloidal solution with the fingermark residue. The conventional MMD method exploits the interaction between the citrate capped GNPs and the amino acids in the fingermark residue. The exact reaction mechanism between this two substrates are still unknown. Hence, a few researches have been conducted to directly target the fingermark residue by using immunological reaction [11-13].

Leggett et al. [14] used antibody functionalised nanoparticle to identify drug metabolites present in fingermark and simultaneously visualizing the mark [14]. The citrate capped GNPs were synthesised using the Turkevich method, which involves single phase waterbased reduction of gold salt by citrate [15]. The citrate acts as a double agent aiding in the reduction of gold salt and also stabilise the formed colloidal gold [15]. The negatively charged citrate ions that caps the GNPs repels one another and in turn, avoid the gold particles from aggregating [16].

Leggett et al. [14] used the Turkevich method to synthesise the GNPs and then modified the GNPs surface with N-succinimidyl 3-(2-pyridyldithio) propionate (SPDP) tagged Protein A. Protein A then facilitates the binding of the anti-cotinine antibody molecule which will detect and bind to cotinine present in the fingermark residue [14]. This method is capable of offering visualisation of fingermark containing cotinine only and its success depends on the homogenous distribution of cotinine along the ridges, or not only partial prints will be obtained [14,17]. Another MMD method combined with immunoassay technique to visualise latent fingermarks was also introduced in the year 2014. The method uses Immunoglobulin G (IgG) to cap the GNPs and subsequently enhanced using silver developer upon deposition onto fingermarks [11].

\section{pH limitation of the MMD method}

The conventional MMD method have strict $\mathrm{pH}$ limitation in order for successful visualisation of the fingermark. $\mathrm{pH}$ has to be maintained between 2.5 to 3.0 during the Aurum deposition phase $[6,7]$. Hitherto only theoretical postulations have been made to explain the nature of reaction between the GNPs in the colloidal gold solution and the residue of fingermark [17]. The information obtained so far to assist these postulations is the $\mathrm{pH}$ at which this interaction occurs. Observation have also been made that bare surface of GNPs are needed for the successful binding of amino acids or proteins to GNP to produce desired outcome [18]. The interaction between GNPs and the amino acids have been arguably elusive, however three interaction possibilities have been narrowed down. The electrostatic interactions between negatively charged GNPs and positively charged amino acids, covalent interaction between the thiol/amine group of the amino acids with the carboxyl terminal of citrate coated GNPs and hydrophobic interactions between amino acids and GNPs are the three speculations that have been made [18].

Electrostatic interaction between the negatively charged citrate capped GNPs and the positively charged N-terminus of the amino acid has been the most popular theory so far. The amino acids are carboxylic acids that has an amine function [19]. There are four categories of amino acids the amino acids with nonpolar side chains, amino acids with polar but non-ionised side chains and amino acids with acidic and basic side chains [19,20]. The 20 amino acids that are coded for DNA directed protein synthesis in the human body are listed in the Table 1 below. Classifications of amino acids Nonpolar amino acids Non-ionised Polar Amino acids Amino acids with basic side chain Amino acids with acidic side chain 
Table 1: List of 20 essential amino acids classified according to their side chains.

\begin{tabular}{|l|c|c|c|c|}
\hline $\begin{array}{c}\text { Classifications of Amino } \\
\text { Acids }\end{array}$ & Nonpolar Amino Acids & $\begin{array}{c}\text { Non-ionised Polar } \\
\text { Amino acids }\end{array}$ & $\begin{array}{c}\text { Amino Acids with } \\
\text { Basic Side Chain }\end{array}$ & $\begin{array}{c}\text { Amino Acids with Acidic } \\
\text { Side Chain }\end{array}$ \\
\hline \multirow{3}{*}{ List of the Amino acids } & Glysine & Asparagine & Lysine & Aspartic acid \\
\cline { 2 - 5 } & Alanine & Glutamine & Arginine & Glutamic acid \\
\cline { 2 - 5 } & Valine & Serine & Histidine & \\
\cline { 2 - 5 } & Leucine & Threonine & & \\
\cline { 2 - 5 } & Isoleucine & Tyrosine & & \\
\cline { 2 - 5 } & Methionine & Cysteine & & \\
\cline { 2 - 5 } & Proline & & & \\
\hline
\end{tabular}

All of the amino acids listed above has pKa1 at the range 1.83 to 2.83 and $\mathrm{pKa} 2$ at the range 8.80 to 10.6020 . The amino acids are known to exist in the zwitterion forms in solution [21]. The carboxyl group of the amino acids are ionised at lower $\mathrm{pH}$ while the positive charge of the amine terminal are maintained, however at higher $\mathrm{pH}$ the positive charge of the amine terminal are neutralised by deprotonation [22]. This means that the amino acids bears a positive charge at the $\mathrm{N}$-terminus until the $\mathrm{pH}$ is raised above the isoelectric point. Hence the aggregation of amino acids and the citrate capped GNPs occurs most optimally at lower $\mathrm{pH}$ when the amino acids bears a positive charge [22].

Another findings reported that the addition of aspartic acid during the synthesis of citrate reduced GNPs increases the range of working $\mathrm{pH}$ for the detection of latent fingermarks [23]. The chemical structure of the aspartic acid contains two carboxyl and one amine side chains $\left(\mathrm{HOOCCH}\left(\mathrm{NH}_{2}\right) \mathrm{CH}_{2} \mathrm{COOH}\right)$ while citrate molecules contains three carboxyl groups [20]. At lower $\mathrm{pH}$, the carboxyl group of the citrate molecules are protonated to reduce the negative charge [6]. Nonetheless, when GNPs are synthesised with both aspartic acid and citric acid the negative charge of the GNPs are strengthened by aspartic acid which has pKa value of 3.820 .

This paper also reported that the $\mathrm{pH}$ range was increased until pH 5 for successful detection6. This shows that the addition of aspartic acid somehow stabilises the reaction between the GNPs and amino acids at higher $\mathrm{pH}$ at which point the positive charge of the amine terminal of amino acids are reduced. This observation brings us to believe that the electrostatic interaction may not be the only interaction involved, perhaps the formation of amide bond between amines in amino acids and the carboxyl group of the capped GNPs is a more likely pathway.

The most recent postulations were supported by the findings of Moret et al. that suggested that the electrostatic interaction may not be the exact reaction that attracts the GNPs to the amino acids. He conducted study using silicon dioxide capped with several molecules that would mimic the MMD method [23]. This study was attempted to strengthen the negative charge of the NPs at low $\mathrm{pH}$ by conjugating succinic anhydride, methyl phosponate and sulfonate molecule onto silicon dioxide nanoparticles (SDNPs). The succinic anhydride that has carboxyl group was able to detect luminescent fingermark at $\mathrm{pH} 3.5$ however the methyl phosponate and sulfanate capped SDNPs was reported to have absolutely no affinity for the fingermark residues [23]. These findings argued that the electrostatic theory is not the interaction mechanism by which the GNPs are attracted to the fingermark residue but the formation of peptide bond between the amine terminal in the amino acids and the carboxyl group in the capping substrate of the GNPs is more likely to be the interaction that aids gold deposition [23].

\section{Conclusion}

Beside the issues addressed above the application of GNPs in the development of latent fingermarks also have other disadvantages that have yet to be explored. The long term stability of the reagents synthesized remains a problem with the MMD technique, where the physical enhancer have to be freshly prepared prior to development process for best results. Other than that, the GNP based wet chemical methods also cannot be used for large objects that are not mobile and an on-site reagent delivery system for immediate visualization still have not yet been conceived. Development of alternative MMD method have improved the shelf life of the reagents, yet, in comparison to powdering method, ninhydrin method or the superglue method are better equipped for forensic applications8. MMD technique still remains as a last resort for fingermark development. Future research maybe directed in improving the applicability of the method in regular investigation process.

\section{References}

1. Schnetz B, Margot P (2001) Technical note: Latent fingermarks, colloidal gold and multimetal deposition (MMD) Optimisation of the method. Forensic Sci Int 118(1): 21-28.

2. Fairley C, Bleay SM, Sears VG, NicDaeid N (2012) A comparison of multi-metal deposition processes utilising gold nanoparticles and an evaluation of their application to "low yield" surfaces for finger mark development. Forensic Sci Int 217(1-3): 5-18. 
3. Choi MJ, McDonagh AM, Maynard P, Roux C (2008) Metal-containing nanoparticles and nano-structured particles in fingermark detection. Forensic Sci Int 179(2-3): 87-97.

4. Dilag J, Kobus J, H, Ellis AV (2011) Nanotechnology as a new tool for fingermark detection: A review. Curr Nanosci 7(2): 153-159.

5. Becue A, Scoundrianos A, Champod C, Margot P (2008) Fingermark detection based on the in situ growth of luminescent nanoparticlestowards a new generation of multimetal deposition. Forensic Sci Int 179(1): 39-43.

6. Becue A, Scoundrianos A, Moret S (2012) Detection of fingermarks by colloidal gold (MMD/SMD) - beyond the $\mathrm{pH} 3$ limit. Forensic Sci Int 219(1-3): 39-49.

7. Becue A, Champod C, Margot P (2007) Use of gold nanoparticles as molecular intermediates for the detection of fingermarks. Forensic Sci Int 168(2-3): 169-76.

8. Stauffer E, Becue A, Singh KV, Thampi KR, Champod C, et al. (2007) Single-metal deposition (SMD) as a latent fingermark enhancement technique: An alternative to multimetal deposition (MMD). Forensic Sci Int 168(1): 5-9.

9. Turkevich J, Stevenson P, Hillier J (1953) The formation of colloidal gold. J Phys Chem 57(7): 670-673.

10. Gao D, Li F, Song J, Xu X, Zhang Q et al. (2009) One step to detect the latent fingermarks with gold nanoparticles. Talanta 80(2): 479-483.

11. He Y, Xu L, Zhu Y, Wei Q Zhang M, et al. (2014) Immunological multimetal deposition for rapid visualization of sweat fingerprints. Angew Chemie Int Ed 53(46): 12609-12612.

12. Van Dam A, Aalders MCG, van de Braak K, Hardy HJJ, Van Leeuwen TG, Lambrechts SAG (2013) Simultaneous labeling of multiple components in a single fingermark. Forensic Sci Int 232(1-3): 173-179.

13. Spindler X, Hofstetter O, McDonagh AM, Roux C, Lennard C (2011) Enhancement of latent fingermarks on non-porous surfaces using anti-L-amino acid antibodies conjugated to gold nanoparticles. Chem Commun (Camb) 47(19): 5602-5604.
14. Leggett R, Lee-Smith EE, Jickells SM, Russell D (2007) "Intelligent" fingerprinting: Simultaneous identification of drug metabolites and individuals by using antibody-functionalized nanoparticles. Angew Chemie Int Ed 46(22): 4100-4103.

15. Kimling J, Maier M, Okenve B, Kotaidis V, Ballot H, et al. (2006) Turkevich method for gold nanoparticle synthesis revisited. J Phys Chem B 110 (32): 15700-15707.

16. DeLong RK, Reynolds CM, Malcolm Y, Schaeffer A, Severs T, et al. (2010) Functionalized gold nanoparticles for the binding, stabilization, and delivery of therapeutic DNA, RNA, and other biological macromolecules. Nanotechnol Sci Appl 3: 53-63.

17. Choi MJ, McBean KE, Wuhrer R, McDonagh AM, Maynard PJ, et al. (2006) Investigation into the binding of gold nanoparticles to fingermarks using scanning electron microscopy. J Forensic Identif 56(1): 24-32.

18. Arvizo RR, Rana S, Miranda OR, Bhattacharya R, Rotello VM, et al. (2011) Mechanism of Anti-angiogenic Property of Gold Nanoparticles: Role of Nanoparticle Size and Surface Charge. Nanomedicine 7(5):580-587.

19. Mathews CK, Appling DR, Anthony-Cahill SJ, van Holde KE (2013) Biochemistry. ( $4^{\text {th }}$ edn), Pearson Canada Inc, Ontario, Toronto, Canada.

20. Carey FA, Giuliano RM (2011) Organic Chemistry. ( $8^{\text {th }}$ edn), Mc-Graw Hill, New York, USA.

21. Sperling RA, Parak WJ (2010) Surface modification, functionalization and bioconjugation of colloidal inorganic nanoparticles. Philos Trans A Math Phys Eng Sci 368(1915): 1333-1383.

22. Mihailescu GH, Olenic L, Pruneanu S, Bratu I, Kasco I (2007) The Effect of $\mathrm{pH}$ on Amino Acids Binding to Gold Nanoparticles. J Optoelectron Adv Mater 9(3): 756-759.

23. Moret S, Bécue A, Champod C (2014) Nanoparticles for fingermark detection: an insight into the reaction mechanism. Nanotechnology 25(42). 